\title{
COPD: Bluteosinophilie spricht für ICS-Nutzen
}

\author{
Inhalative Steroide spielen bei der COPD-Therapie kaum noch eine Rolle. Bei hohen Eosinophilenzahlen \\ im Blut sind sie aber nach wie vor eine gute Option, wie eine Neuauswertung älterer Daten zeigt.
}

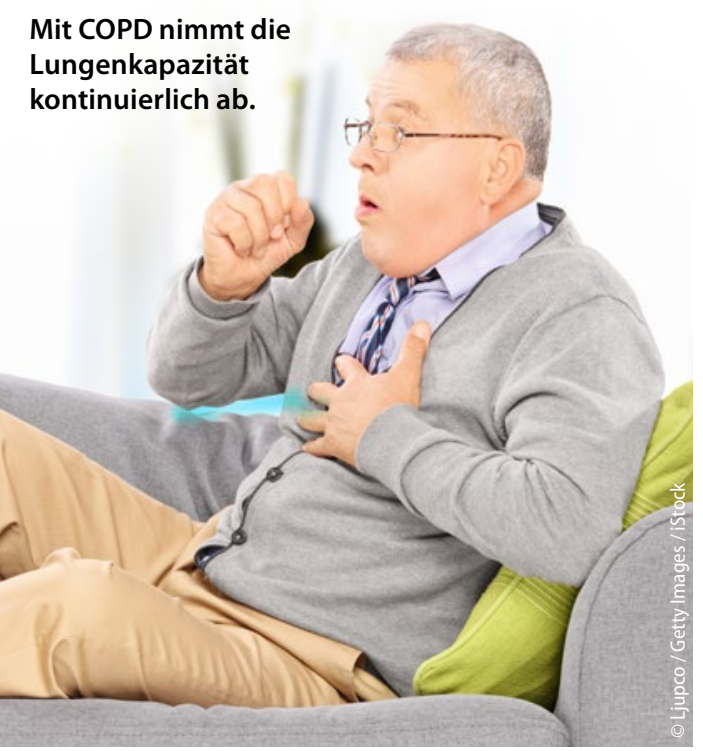

In der im Jahr 2000 publizierten, doppelblinden, placebokontrollierten ISOLDE-Studie mit 751 Patienten mit mäßiger bis schwerer COPD konnte eine Monotherapie mit $2 \times 500 \mu \mathrm{g} / \mathrm{d}$ des inhalativen Kortikosteroids (ICS) Fluticason die jährliche Exazerbationsrate von 1,32 auf 0,99 senken. Ein signifikanter Rückgang der $\mathrm{FEV}_{1}$ ließ sich dagegen nicht feststellen. Auch brachen fast 50\% der Probanden die Teilnahme ab.
Aus anderen Studien ist bekannt, dass das Risiko schwerer Exazerbationen reduziert ist, wenn man die Steroidtherapie so titriert, dass sich die Eosinophilenzahl im Sputum normalisiert. Hohe Eosinophilenzahlen im Sputum waren mit gutem Ansprechen der FEV 1 auf systemische Steroide assoziiert. Leider ist die Bestimmung der Eosinophilen im Sputum technisch schwierig. Daher prüften Statistiker nun anhand der ISOLDE-Daten, ob eine ähnliche Assoziation auch für die Eosinophilen im Blut existiert.

Der jährliche Rückgang der unbeeinflussten $\mathrm{FEV}_{1}$ betrug unter Fluticason $50 \mathrm{ml}$, unter Placebo $59 \mathrm{ml}$. Immerhin war die $\mathrm{FEV}_{1}$ aber nach Anwendung eines Bronchodilatators in der Verumgruppe konstant signifikant höher als in der Placebogruppe.

Ein anderes Bild ergab sich, wenn man die Patienten anhand der Eosinophilenzahl im Blut bei Studienbeginn unterteilte. Bei 456 Patienten lag sie $<2 \%$. In dieser Gruppe gab es keinen Unterschied beim jährlichen Rückgang der $\mathrm{FEV}_{1}$ nach Anwendung eines Bronchodilatators (Fluticason: 54,2 ml, Placebo: $51,3 \mathrm{ml} / \mathrm{Jahr}$ ). Anders sah es bei den 214

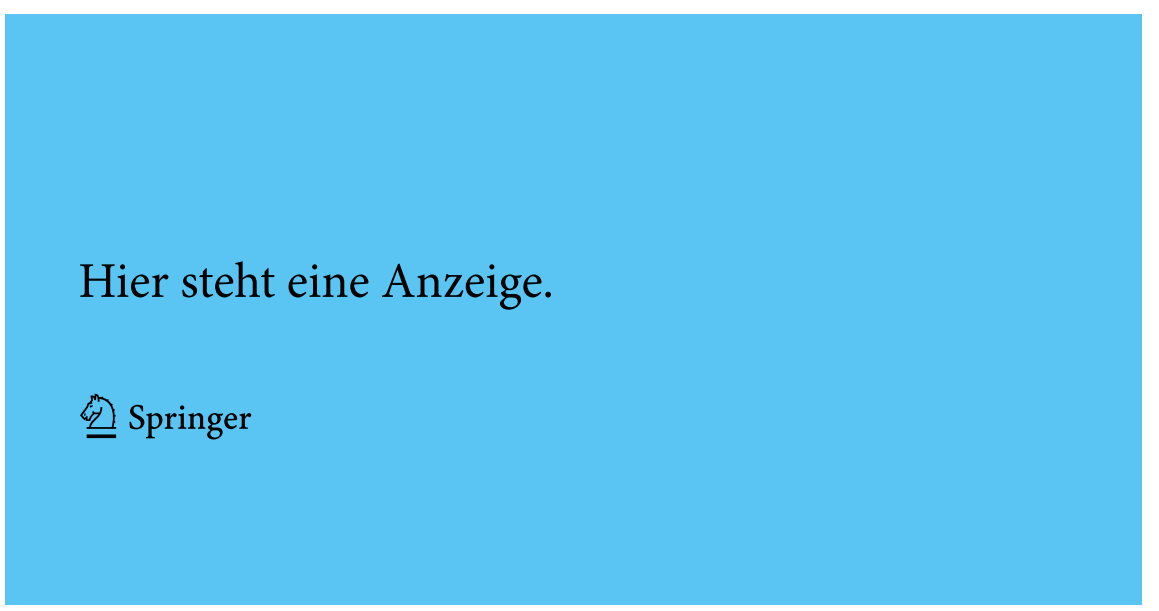

Patienten mit einer Eosinophilenzahl $\geq 2 \%$ aus: Hier verringerte Fluticason den jährlichen Rückgang um 33,9 ml (40,6 vs. $74,5 \mathrm{ml}, \mathrm{p}=0,003)$. Der allgemeine Gesundheitsstatus, erfasst mit gängigen Fragebögen, ging mit Placebo über drei Jahre signifikant stärker zurück als mit Fluticason.

Die Autoren sehen damit ihre These bestätigt, dass COPD-Patienten mit höheren Eosinophilenzahlen eher von inhalativen Steroiden profitieren.

- Barnes NC, Sharma R, Lettis S, Calverley PM. Blood eosinophils as a marker of response to inhaled corticosteroids in COPD. Eur Respir J. 2016;47:1374-82

\section{KOMMENTAR}

In die Therapie der COPD ist in den letzten Jahren erhebliche Dynamik gekommen. Gerade mit der Einführung der Kombinationen aus lang wirksamen Anticholinergika und $\beta_{2}$-Sympathikomimetika sind die inhalativen Steroide weitgehend in den Hintergrund getreten. Mit dem literarischen Hinweis auf die Bedeutung der Eosinophilenzahl für das Ansprechen auf inhalative Steroide haben die Autoren aber einen wichtigen Punkt angesprochen, der bisher bei der Auswahl der richtigen Therapie zu wenig berücksichtigt wurde. Damit liegt die Studie im modernen Trend auf der Suche nach maßgeschneiderten individuellen Therapien.

Möglicherweise befand sich in der ISOLDEStudie in der Gruppe mit höheren Eosinophilenzahlen ein größerer Anteil von Patienten mit sogenannten Overlap-Syndrom - einer Erkrankung, die auch Charakteristika eines Asthma bronchiale aufweist. Dies lässt sich anhand der alten Daten nicht mehr klären. Zumindest kreiert die Beobachtung aber neue Hypothesen, die in neuen prospektiven Studien zur COPD-Therapie beachtet werden sollten. Manchmal ist es in dieser Hinsicht doch ganz nützlich, ältere Daten unter einem neuen Aspekt noch einmal aufzurollen.

Prof. Dr. med. H. S. FüeßI 